\title{
REDUCTIONS OF POINTS ON ALGEBRAIC GROUPS, II
}

\author{
PETER BRUIN \\ Mathematisch Instituut, Universiteit Leiden, Postbus 9512, 2300 RA Leiden, The Netherlands \\ e-mail:P.J.Bruin@math.leidenuniv.nl \\ ANTONELLA PERUCCA \\ Department of Mathematics, University of Luxembourg, 6, Avenue de la Fonte, \\ 4364 Esch-sur-Alzette, Luxembourg \\ e-mail: antonella.perucca@uni.lu
}

(Received 15 October 2019; revised 16 March 2020; accepted 26 June 2020; first published online 28 July 2020)

\begin{abstract}
Let $A$ be the product of an abelian variety and a torus over a number field $K$, and let $m \geqslant 2$ be a square-free integer. If $\alpha \in A(K)$ is a point of infinite order, we consider the set of primes $\mathfrak{p}$ of $K$ such that the reduction $(\alpha \bmod \mathfrak{p})$ is well defined and has order coprime to $m$. This set admits a natural density, which we are able to express as a finite sum of products of $\ell$-adic integrals, where $\ell$ varies in the set of prime divisors of $m$. We deduce that the density is a rational number, whose denominator is bounded (up to powers of $m$ ) in a very strong sense. This extends the results of the paper Reductions of points on algebraic groups by Davide Lombardo and the second author, where the case $m$ prime is established. $11 \mathrm{G} 10$

2010 Mathematics Subject Classification. Primary: 11F80; Secondary: 14L10, 11G05,
\end{abstract}

1. Introduction. This article is the continuation of the paper Reductions of points on algebraic groups by Davide Lombardo and the second author [4]. We refer to this other work for the history of the problem, which started in the 1960s with work of Hasse on the multiplicative orders of rational numbers modulo primes.

Let $A$ be the product of an abelian variety and a torus over a number field $K$, and let $m \geqslant 2$ be a square-free integer. If $\alpha \in A(K)$ is a point of infinite order, we consider the set of primes $\mathfrak{p}$ of $K$ such that the reduction $(\alpha \bmod \mathfrak{p})$ is well defined and has order coprime to $m$. This set admits a natural density (see Theorem 7), which we denote by $\operatorname{Dens}_{m}(\alpha)$.

The main question is whether we can write

$$
\operatorname{Dens}_{m}(\alpha)=\prod_{\ell} \operatorname{Dens}_{\ell}(\alpha)
$$

where $\ell$ varies over the prime divisors of $m$. Let $K(A[m])$ be the $m$-torsion field of $A$. We prove that (1.1) holds if $K(A[m])=K$ (i.e. if $A(K)$ contains all $m$-torsion points) or, more generally, if the degree $[K(A[\ell]): K]$ is a power of $\ell$ for every prime divisor $\ell$ of $m$ (see Corollary 18). Indeed, (1.1) holds if the torsion fields/Kummer extensions of $\alpha$ related to different prime divisors of $m$ are linearly disjoint over $K$. In general, (1.1) does not hold: see Section 7.2 for an explicit example.

We are able to express Dens ${ }_{m}(\alpha)$ as an integral over the image of the $m$-adic representation (see Theorem 16) and also as a finite sum of products of $\ell$-adic integrals (see 
Theorem 19). The latter decomposition allows us to prove that $\operatorname{Dens}_{m}(\alpha)$ is a rational number whose denominator is uniformly bounded in a very strong sense (see Corollary 20).

Finally, we study Serre curves in detail in Section 6. With the partition given in Section 6.3, one can very easily compute $\operatorname{Dens}_{m}(\alpha)$ if the $m^{n}$-Kummer extensions of $\alpha$ (defined in Section 3) have maximal degree for all $n$ or, more generally, if the degrees of these extensions are known and are the same with respect to the base fields $K$ and $K(A[m])$.

In general, to compute the density $\operatorname{Dens}_{m}(\alpha)$ for the product of an abelian variety and a torus, we only need information on the Galois group of the $m^{n}$-torsion fields/Kummer extensions of $\alpha$ for some sufficiently large $n$. Thus, a theoretical algorithm to compute the density exists, because the growth in $n$ of the $m^{n}$-torsion fields/Kummer extensions of $\alpha$ is eventually maximal (see Proposition 5 and Remark 6 in view of [4, Lemma 11]).

Finally, we point out that since the category of algebraic groups that we consider is stable under products, our results allow us to replace $\alpha$ by a finitely generated subgroup of $A(K)$; see Remark 22.

2. Integration on profinite groups. For every profinite group $G$, we write $\mu_{G}$ for the normalised Haar measure on $G$. More generally, if $X$ is a $G$-torsor, we write $\mu_{X}$ for the normalised Haar measure on $X$, defined by transporting $\mu_{G}$ along any isomorphism $G \cong X$ of $G$-torsors.

LEMMA 1. Let $G$ be a profinite group, and let $H$ be an open subgroup of $G$. Suppose that we have $G=\prod_{\ell} G_{\ell}$, where $\ell$ varies in a finite set of prime numbers, and each $G_{\ell}$ is a profinite group containing a pro- $\ell$-group $G_{\ell}^{\prime}$ as an open subgroup. Let $G^{\prime}=\prod_{\ell} G_{\ell}^{\prime}$ and $H^{\prime}=H \cap G^{\prime}$. For each $x \in H / H^{\prime}$, let $H(x)$ be the fibre over $x$ of the quotient map $H \rightarrow H / H^{\prime}$.

(1) The subgroup $H^{\prime}$ is open in $H$, and for each $x \in H / H^{\prime}$, the normalised Haar measure on the $H^{\prime}$-torsor $H(x)$ is

$$
\mu_{H(x)}=\left.\left(H: H^{\prime}\right) \mu_{H}\right|_{H(x)} .
$$

(2) We can write

$$
H^{\prime}=\prod_{\ell} H_{\ell}^{\prime}
$$

where each $H_{\ell}^{\prime}$ is a pro- $\ell$-group, and the normalised Haar measures on $H^{\prime}$ and the $H_{\ell}^{\prime}$ are related by

$$
\mu_{H^{\prime}}=\prod_{\ell} \mu_{H_{\ell}^{\prime}}
$$

(3) We can write the $H^{\prime}$-torsor $H(x)$ as

$$
H(x)=\prod_{\ell} H_{\ell}(x)
$$

where each $H_{\ell}(x)$ is a $H_{\ell}^{\prime}$-torsor, and the normalised Haar measures on $H(x)$ and the $H_{\ell}(x)$ are related by

$$
\mu_{H(x)}=\prod_{\ell} \mu_{H_{\ell}(x)}
$$

Proof. The claim that $H^{\prime}$ is open in $H$ holds because $G^{\prime}$ is open in $G$. The measure $\left.\mu_{H}\right|_{H(x)}$ is $H^{\prime}$-invariant and satisfies $\int_{H(x)} \mu_{H}=\frac{1}{\left(H: H^{\prime}\right)}$; this proves (1). Because $G^{\prime}$ is a 
product of pro- $\ell$-groups for pairwise different $\ell$, every closed subgroup of $G^{\prime}$ is similarly a product of pro- $\ell$-groups. This shows the existence of the $H_{\ell}^{\prime}$ as in (2); the claim about $\mu_{H^{\prime}}$ follows because $\prod_{\ell} \mu_{H_{\ell}^{\prime}}$ satisfies the properties of the normalised Haar measure on $H^{\prime}$. Finally, (3) is proved in the same way as (2).

Proposition 2. With the notation of Lemma 1, let $f: H \rightarrow \mathbb{C}$ be an integrable function.

(1) We have

$$
\int_{H} f d \mu_{H}=\frac{1}{\left(H: H^{\prime}\right)} \sum_{x \in H / H^{\prime}} \int_{H(x)} f d \mu_{H(x)} .
$$

(2) Suppose that for each $x \in H / H^{\prime}$, the restriction of $f$ to $H(x)$ admits a product decomposition

$$
\left.f\right|_{H(x)}=\prod_{\ell} f_{x, \ell}
$$

where the $f_{x, \ell}: H_{\ell}(x) \rightarrow \mathbb{C}$ are integrable functions. Then we have

$$
\int_{H} f d \mu_{H}=\frac{1}{\left(H: H^{\prime}\right)} \sum_{x \in H / H^{\prime}} \prod_{\ell} \int_{H_{\ell}(x)} f_{x, \ell} d \mu_{H_{\ell}(x)} .
$$

Proof. Part (1) follows by rewriting $\int_{H} f d \mu_{H}$ as $\sum_{x \in H / H^{\prime}} \int_{H(x)} f d \mu_{H}$ and applying Lemma 1(1). Part (2) follows from part (1), Lemma 1(3) and the assumption on $f$.

3. The arboreal representation. Let $K$ be a number field, and let $\bar{K}$ be an algebraic closure of $K$. Let $A$ be a connected commutative algebraic group over $K$, and let $b_{A}$ be the first Betti number of $A$. We fix a square-free integer $m \geqslant 2$. Below, we let $\ell$ vary in the set of prime divisors of $m$. We also fix a point $\alpha \in A(K)$.

We define $T_{m} A$ as the projective limit of the torsion groups $A\left[m^{n}\right]$ for $n \geqslant 1$; we can write $T_{m} A=\prod_{\ell} T_{\ell} A$, where the Tate module $T_{\ell} A$ is a free $\mathbb{Z}_{\ell}$-module of rank $b_{A}$.

We define the torsion fields

$$
K_{m^{-n}}:=K\left(A\left[m^{n}\right]\right) \text { for } n \geqslant 1
$$

and

$$
K_{m^{-\infty}}:=\bigcup_{n \geqslant 1} K_{m^{-n}}
$$

The Galois action on the $m$-power torsion points of $A$ gives the $m$-adic representation of $A$, which maps $\operatorname{Gal}(\bar{K} / K)$ to the automorphism group of $T_{m} A$. We can also speak of the mod $m^{n}$ representation, which describes the Galois action on $A\left[m^{n}\right]$. Choosing a $\mathbb{Z}$-basis for $T_{\ell} A$ for every prime divisor $\ell$ of $m$, we can identify the image of the $m$-adic representation with a subgroup of $\prod_{\ell} \mathrm{GL}_{b_{A}}\left(\mathbb{Z}_{\ell}\right)$ and the image of the mod $m^{n}$ representation with a subgroup of $\prod_{\ell} \mathrm{GL}_{b_{A}}\left(\mathbb{Z} / \ell^{n} \mathbb{Z}\right)$.

For $n \geqslant 1$, let $m^{-n} \alpha$ be the set of points in $A(\bar{K})$ whose $m^{n}$ th multiple equals $\alpha$. We also write

$$
m^{-\infty} \alpha=\lim _{n \geqslant 1} m^{-n} \alpha
$$


This is the set of sequences $\beta=\left\{\beta_{n}\right\}_{n \geqslant 1}$ such that $m \beta_{1}=\alpha$ and $m \beta_{n+1}=\beta_{n}$ for every $n \geqslant 1$; it is a torsor under $T_{m} A$. We note that $m^{-n} 0=A\left[m^{n}\right]$ and $m^{-\infty} 0=T_{m} A$.

We define the fields

$$
K_{m^{-n} \alpha}:=K\left(m^{-n} \alpha\right) \text { for } n \geqslant 1
$$

and

$$
K_{m^{-\infty} \alpha}:=\bigcup_{n \geqslant 1} K_{m^{-n} \alpha} .
$$

We call the field extension $K_{m^{-n} \alpha} / K_{m^{-n}}$ the $m^{n}$-Kummer extension defined by the point $\alpha$. We view the $m$-adic representation as a representation of $\operatorname{Gal}\left(K_{m^{-\infty} \alpha} / K\right)$.

We fix an element $\beta \in m^{-\infty} \alpha$ and define the arboreal representation

$$
\begin{aligned}
\omega_{\alpha, m^{\infty}}: \operatorname{Gal}\left(K_{m^{-\infty} \alpha} / K\right) & \longrightarrow T_{m} A \rtimes \operatorname{Aut}\left(T_{m} A\right) \\
\sigma & \longmapsto(t, M),
\end{aligned}
$$

where $M$ is the image of $\sigma$ under the $m$-adic representation and $t=\sigma(\beta)-\beta$. Then, $\omega_{\alpha, m^{\infty}}$ is an injective homomorphism of profinite groups identifying $\operatorname{Gal}\left(K_{m^{-\infty}} / K\right)$ with a subgroup of

$$
T_{m} A \rtimes \operatorname{Aut}\left(T_{m} A\right) \cong \prod_{\ell} \mathbb{Z}_{\ell}^{b_{A}} \rtimes \prod_{\ell} \operatorname{GL}_{b_{A}}\left(\mathbb{Z}_{\ell}\right) \cong \prod_{\ell}\left(\mathbb{Z}_{\ell}^{b_{A}} \rtimes \mathrm{GL}_{b_{A}}\left(\mathbb{Z}_{\ell}\right)\right) .
$$

Likewise, for each $n \geqslant 1$, the choice of $\beta$ defines a homomorphism

$$
\begin{aligned}
\omega_{\alpha, m^{n}}: \operatorname{Gal}\left(K_{m^{-n} \alpha} / K\right) & \longrightarrow A\left[m^{n}\right] \rtimes \operatorname{Aut}\left(A\left[m^{n}\right]\right) \\
\sigma & \longmapsto(t, M),
\end{aligned}
$$

where $t$ and $M$ are defined in a similar way as above. This identifies $\operatorname{Gal}\left(K_{m^{-n} \alpha} / K\right)$ with a subgroup of

$$
A\left[m^{n}\right] \rtimes \operatorname{Aut}\left(A\left[m^{n}\right]\right) \cong \prod_{\ell}\left(\left(\mathbb{Z} / \ell^{n} \mathbb{Z}\right)^{b_{A}} \rtimes \mathrm{GL}_{b_{A}}\left(\mathbb{Z} / \ell^{n} \mathbb{Z}\right)\right) .
$$

We denote by $\mathcal{G}\left(\ell^{\infty}\right)$ the image of the $\ell$-adic representation in $\operatorname{Aut}\left(T_{\ell} A\right) \cong \mathrm{GL}_{b_{A}}\left(\mathbb{Z}_{\ell}\right)$ and by $\mathcal{G}\left(\ell^{n}\right)$ the image of the $\bmod \ell^{n}$ representation in $\operatorname{Aut}\left(A\left[\ell^{n}\right]\right) \cong \mathrm{GL}_{b_{A}}\left(\mathbb{Z} / \ell^{n} \mathbb{Z}\right)$. Similarly, we denote by $\mathcal{G}\left(m^{\infty}\right)$ the image of the $m$-adic representation $\operatorname{in} \operatorname{Aut}\left(T_{m} A\right) \cong$ $\prod_{\ell} \mathrm{GL}_{b_{A}}\left(\mathbb{Z}_{\ell}\right)$ and by $\mathcal{G}\left(m^{n}\right)$ the image of the $\bmod m^{n}$ representation in $\operatorname{Aut}\left(A\left[m^{n}\right]\right) \cong$ $\mathrm{GL}_{b_{A}}\left(\mathbb{Z} / m^{n} \mathbb{Z}\right)$.

We write $d_{A, \ell}$ for the dimension of the Zariski closure of $\mathcal{G}\left(\ell^{\infty}\right)$ in $\mathrm{GL}_{b_{A}, \mathbb{Q}_{\ell}}$, and we put

$$
D_{A, m}=\prod_{\ell \mid m} \ell^{d_{A, \ell}} .
$$

We note that the $d_{A, \ell}$ and $D_{A, m}$ do not change when replacing $K$ by a finite extension. Moreover, assuming the Mumford-Tate conjecture, all $d_{A, \ell}$ are equal to $d_{A}$, the dimension of the Mumford-Tate group, implying $D_{A, m}=m^{d_{A}}$. This is known, for example, when $A$ is an elliptic curve; in this case, $d_{A}$ equals 2 if $A$ has complex multiplication, and 4 otherwise.

DEFINITION 3. We say that $(A / K, m)$ satisfies eventual maximal growth of the torsion fields if there exists a positive integer $n_{0}$ such that for all $N \geqslant n \geqslant n_{0}$ we have

$$
\left[K_{m^{-N}}: K_{m^{-n}}\right]=D_{A, m}^{N-n} .
$$


We say that $(A / K, m, \alpha)$ satisfies eventual maximal growth of the Kummer extensions if there exists a positive integer $n_{0}$ such that for all $N \geqslant n \geqslant n_{0}$ we have

$$
\left[K_{m^{-N} \alpha}: K_{m^{-n} \alpha}\right]=\left(m^{b_{A}} D_{A, m}\right)^{N-n} .
$$

REMARK 4. Condition (3.1) means that there is eventual maximal growth of the torsion fields, that $K_{m^{-n} \alpha}$ and $K_{m^{-N}}$ are linearly disjoint over $K_{m^{-n}}$ and that we have

$$
\left[K_{m^{-N} \alpha}: K_{m^{-N}}\left(m^{-n} \alpha\right)\right]=m^{b_{A}(N-n)} .
$$

If there is eventual maximal growth of the Kummer extensions, the rational number

$$
\mathrm{C}_{m}:=m^{b_{A} n} /\left[K_{m^{-n} \alpha}: K_{m^{-n}}\right]
$$

is independent of $n$ for $n \geqslant n_{0}$. In fact, $C_{m}$ is an integer because $\omega_{\alpha, m^{n}}$ maps $\operatorname{Gal}\left(K_{m^{-n} \alpha} / K_{m^{-n}}\right)$ injectively into $A\left[m^{n}\right] \cong(\mathbb{Z} / m \mathbb{Z})^{b_{A}}$.

Proposition 5. If $A$ is a semiabelian variety, then $(A / K, m)$ satisfies eventual maximal growth of the torsion fields. If $A$ is the product of an abelian variety and a torus and $\mathbb{Z} \alpha$ is Zariski dense in $A$, then $(A / K, m, \alpha)$ satisfies eventual maximal growth of the Kummer extensions.

Proof. By [4, Lemma 12], if $A$ is a semiabelian variety and $\ell$ is a prime divisor of $m$, then $(A / K, \ell, \alpha)$ satisfies eventual maximal growth of the torsion fields. We also know that the degree $\left[K_{\ell^{-n}}: K_{\ell^{-1}}\right]$ is a power of $\ell$ for each $n$. Therefore, the extensions $K_{m^{-1}} K_{\ell^{-n}}$ for $\ell \mid m$ are linearly disjoint over $K_{m^{-1}}$ and the first assertion follows. By [4, Remark 9], the second assertion holds for $(A / K, \ell, \alpha)$, where $\ell$ is any prime divisor of $m$. We conclude because the degrees of these Kummer extensions are powers of $\ell$.

\section{Relating the density and the arboreal representation.}

4.1. The existence of the density. Let $(A / K, m, \alpha)$ be as in Section 3. From now on, we assume that $(A / K, m, \alpha)$ satisfies eventual maximal growth of the Kummer extensions.

REMARK 6. This is not a restriction if $A$ is the product of an abelian variety and a torus by Proposition 5. Indeed, consider the number of connected components of the Zariski closure of $\mathbb{Z} \alpha$. If this number is not coprime to $m$, then the density $\operatorname{Dens}_{m}(\alpha)$ is zero by [5, Main Theorem] while if it is coprime to $m$ we may replace $\alpha$ by a multiple to reduce to the case where the Zariski closure of $\mathbb{Z} \alpha$ is connected. Finally, we may replace $A$ by the Zariski closure of $\mathbb{Z} \alpha$ and reduce to the case where $\mathbb{Z} \alpha$ is Zariski dense. Also notice that if $A$ is simple (i.e. has exactly two connected algebraic subgroups), then eventual maximal growth of the Kummer extensions is satisfied as soon as $\alpha$ has infinite order.

The $T_{m} A$-torsor $m^{-\infty} \alpha$ from Section 3 defines a Galois cohomology class

$$
C_{\alpha} \in H^{1}\left(\operatorname{Gal}\left(K_{m^{-\infty} \alpha} / K\right), T_{m} A\right) .
$$

For any choice of $\beta \in m^{-\infty} \alpha$, this is the class of the cocycle

$$
\begin{aligned}
c_{\beta}: \operatorname{Gal}\left(K_{m^{-\infty}} / K\right) & \longrightarrow \\
\sigma & T_{m} A \\
\sigma & \longmapsto \sigma(\beta)-\beta .
\end{aligned}
$$


We also consider the restriction map with respect to the cyclic subgroup generated by some element $\sigma \in \operatorname{Gal}\left(K_{m^{-\infty} \alpha} / K\right)$ :

$$
\operatorname{Res}_{\sigma}: H^{1}\left(\operatorname{Gal}\left(K_{m^{-\infty}} / K\right), T_{m} A\right) \longrightarrow H^{1}\left(\langle\sigma\rangle, T_{m} A\right) .
$$

THEOREM 7. If $(A / K, m, \alpha)$ satisfies eventual maximal growth of the Kummer extensions, then the density $\operatorname{Dens}_{m}(\alpha)$ exists and equals the normalised Haar measure in $\operatorname{Gal}\left(K_{m^{-\infty} \alpha} / K\right)$ of the subset

$$
\begin{aligned}
S_{\alpha} & :=\left\{\sigma \in \operatorname{Gal}\left(K_{m^{-\infty} \alpha} / K\right) \mid C_{\alpha} \in \operatorname{ker}\left(\operatorname{Res}_{\sigma}\right)\right\} \\
& =\left\{\sigma \in \operatorname{Gal}\left(K_{m^{-\infty} \alpha} / K\right) \mid \sigma(\beta)=\beta \text { for some } \beta \in m^{-\infty} \alpha\right\} .
\end{aligned}
$$

Proof. The generalisations of [2, Theorem 3.2] and [4, Theorem 7] to the composite case are straightforward.

Similarly to [4, Remark 21], we may equivalently consider $S_{\alpha}$ as a subset of either $\operatorname{Gal}(\bar{K} / K)$ or $\operatorname{Gal}\left(K_{m^{-\infty} \alpha} / K\right)$ with their respective normalised Haar measures.

Proposition 8. If $L / K$ is any Galois extension that is linearly disjoint from $K_{m^{-\infty} \alpha}$ over $K$, then we have $\operatorname{Dens}_{L}(\alpha)=\operatorname{Dens}_{K}(\alpha)$.

Proof. The generalisation of [4, Proposition 22] to the composite case is straightforward.

4.2. Counting elements in the image of the arboreal representation. By Theorem 7, computing Dens $s_{m}(\alpha)$ comes down to computing the Haar measure of $S_{\alpha}$ in $\operatorname{Gal}\left(K_{m^{-\infty} \alpha} / K\right)$. This is why we now investigate the Galois groups $\operatorname{Gal}\left(K_{m^{-n} \alpha} / K\right)$ for positive integers $n$.

For $M \in \mathcal{G}\left(m^{n}\right)$ we define

$$
\mathcal{W}_{m^{n}}(M):=\left\{t \in A\left[m^{n}\right] \mid(t, M) \in \operatorname{Gal}\left(K_{m^{-n} \alpha} / K\right)\right\}
$$

and

$$
\mathrm{W}_{m^{n}}(M):=\frac{\#\left(\operatorname{Im}(M-I) \cap \mathcal{W}_{m^{n}}(M)\right)}{\# \operatorname{Im}(M-I)} \in \mathbb{Q} .
$$

We note that $\mathcal{W}_{m^{n}}(M)$ is a $\operatorname{Gal}\left(K_{m^{-n} \alpha} / K_{m^{-n}}\right)$-torsor and in particular satisfies

$$
\# \mathcal{W}_{m^{n}}(M)=\left[K_{m^{-n} \alpha}: K_{m^{-n}}\right] .
$$

For every prime divisor $\ell$ of $m$ and every $n \geqslant 1$, we consider the Galois group of the compositum $K_{\ell^{-n} \alpha} K_{m^{-1}}$ over $K$ and the inclusion

$$
\iota_{\alpha, \ell^{n}}: \operatorname{Gal}\left(K_{\ell^{-n} \alpha} K_{m^{-1}} / K\right) \hookrightarrow\left(A\left[\ell^{n}\right] \rtimes \mathcal{G}\left(\ell^{n}\right)\right) \times \mathcal{G}(m) .
$$

For all $x \in \mathcal{G}(m)$ and $V \in \mathcal{G}\left(\ell^{n}\right)$, we define

$$
\mathcal{W}_{x, \ell^{n}}(V):=\left\{\tau \in A\left[\ell^{n}\right] \mid(\tau, V, x) \in \operatorname{Im} \iota_{\alpha, \ell^{n}}\right\}
$$

and

$$
\mathrm{W}_{x, \ell^{n}}(V):=\frac{\#\left(\operatorname{Im}(V-I) \cap \mathcal{W}_{x, \ell^{n}}(V)\right)}{\# \operatorname{Im}(V-I)} \in \mathbb{Z}[1 / \ell] .
$$

We denote by $\pi_{*}$ the projection onto $\mathcal{G}(*)$. 
Proposition 9. If $x \in \mathcal{G}(m)$ and $M \in \mathcal{G}\left(m^{n}\right)$ are such that $\pi_{m} M=x$, then we have

$$
\mathcal{W}_{m^{n}}(M)=\prod_{\ell} \mathcal{W}_{x, \ell^{n}}\left(\pi_{\ell^{n}} M\right)
$$

and

$$
\mathrm{w}_{m^{n}}(M)=\prod_{\ell} \mathrm{w}_{x, \ell^{n}}\left(\pi_{\ell^{n}} M\right) .
$$

Proof. Since the extensions $K_{\ell^{-n} \alpha} K_{m^{-1}} / K_{m^{-1}}$ have pairwise coprime degrees and hence are linearly disjoint, giving an element of $\operatorname{Gal}\left(K_{m^{-n} \alpha} / K\right)$ mapping to $x \in \mathcal{G}(m)$ is equivalent to giving, for each prime $\ell \mid n$, an element of $\operatorname{Gal}\left(K_{\ell^{-n} \alpha} K_{m^{-1}} / K\right)$ mapping to $x$. Hence, given an element $t=\sum_{\ell} t_{\ell}$ in $A\left[m^{n}\right]=\bigoplus_{\ell} A\left[\ell^{n}\right]$, we have $t \in \mathcal{W}_{m^{n}}(M)$ if and only if for every $\ell$ we have $t_{\ell} \in \mathcal{W}_{x, \ell^{n}}\left(\pi_{\ell^{n}} M\right)$. Therefore $(t, M)$ is in $\operatorname{Gal}\left(K_{m^{-n} \alpha} / K\right)$ if and only if $\left(t_{\ell}, \pi_{\ell^{n}} M, x\right)$ is in the image of $\iota_{\alpha, \ell^{n}}$ for all $\ell$. This implies the first claim. The second claim follows because we have $\operatorname{Im}(M-I)=\bigoplus_{\ell} \operatorname{Im}\left(\pi_{\ell^{n}} M-I\right)$.

LEMma 10. For all $x \in \mathcal{G}(m)$ and $V \in \mathcal{G}\left(\ell^{\infty}\right)$, the value $\mathrm{w}_{x, \ell^{n}}(V)$ is constant for $n$ sufficiently large.

Proof. This is proved as in [4, Lemma 25].

By Lemma 10, we can define

$$
\mathrm{w}_{x, \ell^{\infty}}(V)=\lim _{n \rightarrow \infty} \mathrm{w}_{x, \ell^{n}}(V) \in \mathbb{Z}[1 / \ell] .
$$

From Proposition 9 we deduce that for all $M \in \mathcal{G}\left(m^{\infty}\right)$, the value $\mathrm{w}_{m^{n}}(M)$ is also constant for $n$ sufficiently large, so we can analogously define

$$
\mathrm{W}_{m^{\infty}}(M)=\lim _{n \rightarrow \infty} \mathrm{w}_{m^{n}}(M) \in \mathbb{Q} .
$$

Proposition 11. If $M \in \mathcal{G}\left(m^{\infty}\right)$ is such that $\pi_{m} M=x$, then we have

$$
\mathrm{w}_{m^{\infty}}(M)=\prod_{\ell} \mathrm{w}_{x, \ell^{\infty}}\left(\pi_{\ell \infty} M\right) .
$$

Proof. Taking the limit as $n \rightarrow \infty$ in Proposition 9 yields the claim.

The following lemma gives sufficient conditions for the sets $\mathcal{W}_{m^{n}}(M)$ and the functions $\mathrm{w}_{m^{n}}(M)$ and $\mathrm{w}_{m^{\infty}}(M)$ to admit product decompositions without a dependence on the element $x \in \mathcal{G}(m)$. It will not be used in the remainder of this article.

LEMMA 12. For all primes $\ell \mid m$ and all $n \geqslant 1$, the following conditions are equivalent:

(1) The intersection of the fields $K_{m^{-1}}$ and $K_{\ell^{-n} \alpha}$ is contained in $K_{\ell^{-n}}$.

(2) The intersection of the fields $K_{m^{-1}} K_{\ell^{-n}}$ and $K_{\ell^{-n} \alpha}$ equals $K_{\ell^{-n}}$.

(3) The fields $K_{m^{-1}} K_{\ell^{-n}}$ and $K_{\ell^{-n} \alpha}$ are linearly disjoint over $K_{\ell^{-n}}$.

(4) We have $\left[K_{m^{-1}} K_{\ell^{-n} \alpha}: K_{m^{-1}} K_{\ell^{-n}}\right]=\left[K_{\ell^{-n} \alpha}: K_{\ell^{-n}}\right]$.

(5) We have $\left[K_{m^{-n}} K_{\ell^{-n} \alpha}: K_{m^{-n}}\right]=\left[K_{\ell^{-n} \alpha}: K_{\ell^{-n}}\right]$.

If these conditions are satisfied for all primes $\ell \mid m$ and all $n \geqslant 1$, then the following statements hold:

(6) We have $\mathrm{C}_{m}=\prod_{\ell} \mathrm{C}_{\ell}$.

(7) For all $n \geqslant 1$ and all $M \in \mathcal{G}\left(m^{n}\right)$ we have $\mathcal{W}_{m^{n}}(M)=\prod_{\ell} \mathcal{W}_{\ell^{n}}\left(\pi_{\ell^{n}} M\right)$. 
(8) For all $n \geqslant 1$ and all $M \in \mathcal{G}\left(m^{n}\right)$ we have $\mathrm{w}_{m^{n}}(M)=\prod_{\ell} \mathrm{w}_{\ell^{n}}\left(\pi_{\ell^{n}} M\right)$.

(9) For all $M \in \mathcal{G}\left(m^{\infty}\right)$ we have $\mathrm{w}_{m^{\infty}}(M)=\prod_{\ell} \mathrm{w}_{\ell^{\infty}}\left(\pi_{\ell^{\infty}} M\right)$.

Proof. The equivalence of the conditions (1)-(4) follows from Galois theory, using the fact that all the fields involved are Galois extensions of $K$. The conditions (4) and (5) are equivalent because $\left[K_{m^{-1}} K_{\ell^{-n} \alpha}: K_{m^{-1}} K_{\ell^{-n}}\right]$ is a power of $\ell$ and $\left[K_{m^{-n}}: K_{m^{-1}} K_{\ell^{-n}}\right]$ is prime to $\ell$. If condition (5) holds for a given $n \geqslant 1$ and all primes $\ell \mid m$, then we have

$$
\begin{aligned}
{\left[K_{m^{-n} \alpha}: K_{m^{-n}}\right] } & =\prod_{\ell}\left[K_{m^{-n}} K_{\ell^{-n} \alpha}: K_{m^{-n}}\right] \\
& =\prod_{\ell}\left[K_{\ell^{-n} \alpha}: K_{\ell^{-n}}\right] .
\end{aligned}
$$

This implies that if (5) is true for all primes $\ell \mid m$ and all $n \geqslant 1$, then (6) and (7) hold. Finally, it is clear that (7) implies (8) and (9).

4.3. Partitioning the image of the $\boldsymbol{m}$-adic representation. We view elements of $\mathcal{G}\left(m^{\infty}\right)$ as automorphisms of $A\left[m^{\infty}\right]=\bigcup_{n \geqslant 1} A\left[m^{n}\right]$. We then classify elements $M \in \mathcal{G}\left(m^{\infty}\right)$ according to the group structure of $\operatorname{ker}(M-I)$ and according to the projection $\pi_{m}(M) \in$ $\mathcal{G}(m)$. Note that if $\operatorname{ker}(M-I)$ is finite, then it is a product over the primes $\ell \mid m$ of finite abelian $\ell$-groups that have at most $b_{A}$ cyclic components.

Let $\mathrm{F}$ be a group of the form $\prod_{\ell \mid m} \mathrm{~F}_{\ell}$, where $\mathrm{F}_{\ell}$ is a finite abelian $\ell$-group with at most $b_{A}$ cyclic components. We define the set

$$
\mathcal{M}_{\mathrm{F}}:=\left\{M \in \mathcal{G}\left(m^{\infty}\right) \mid \operatorname{ker}\left(M-I: A\left[m^{\infty}\right] \rightarrow A\left[m^{\infty}\right]\right) \cong \mathrm{F}\right\},
$$

and for every $x \in \mathcal{G}(m)$ we define the set

$$
\mathcal{M}_{x, \mathrm{~F}}:=\left\{M \in \mathcal{G}\left(m^{\infty}\right) \mid \operatorname{ker}\left(M-I: A\left[m^{\infty}\right] \rightarrow A\left[m^{\infty}\right]\right) \cong \mathrm{F}, \pi_{m}(M)=x\right\} .
$$

We denote by $\mathcal{M}_{\mathrm{F}}(*)$ and $\mathcal{M}_{x, \mathrm{~F}}(*)$, respectively, the images of these sets under the reduction map $\mathcal{G}\left(m^{\infty}\right) \rightarrow \mathcal{G}(*)$. We also write

$$
\mathcal{M}:=\bigcup_{\mathrm{F}} \mathcal{M}_{\mathrm{F}}=\bigcup_{x, \mathrm{~F}} \mathcal{M}_{x, \mathrm{~F}},
$$

the union being taken over all $x \in \mathcal{G}(m)$ and over all groups $\mathrm{F}=\prod_{\ell} \mathrm{F}_{\ell}$ as above, up to isomorphism.

PROPOSITION 13. The following holds:

(1) The sets $\mathcal{M}_{x, \mathrm{~F}}$ are measurable in $\mathcal{G}\left(m^{\infty}\right)$, and the set $\mathcal{M}$ of (4.8) is measurable in $\mathcal{G}\left(m^{\infty}\right)$.

(2) If $n>v_{\ell}(\exp F)$ for all $\ell \mid m$, then we have

$$
\mu_{\mathcal{G}\left(m^{\infty}\right)}\left(\mathcal{M}_{x, \mathrm{~F}}\right)=\mu_{\mathcal{G}\left(m^{n}\right)}\left(\mathcal{M}_{x, \mathrm{~F}}\left(m^{n}\right)\right) .
$$

(3) We have $\mu_{\mathcal{G}\left(m^{\infty}\right)}\left(\mathcal{M}_{x, \mathrm{~F}}\right)=0$ if and only if $\mathcal{M}_{x, \mathrm{~F}}=\emptyset$.

(4) If $(A / K, m)$ satisfies eventual maximal growth of the torsion fields, then we have

$$
\mu_{\mathcal{G}\left(m^{\infty}\right)}(\mathcal{M})=1 .
$$

Proof. This is proved as in [4, Lemma 23]. 
5. The density as an integral. Suppose that $(A / K, m, \alpha)$ satisfies eventual maximal growth of the Kummer extensions. Recall from Remark 6 that this is not a restriction if $A$ is the product of an abelian variety and a torus. By Theorem 7, computing Dens ${ }_{m}(\alpha)$ comes down to computing the Haar measure of $S_{\alpha}$ in $\operatorname{Gal}\left(K_{m^{-\infty} \alpha} / K\right)$. The generalisation of [4, Remark 19] to the composite case gives

$$
S_{\alpha}=\left\{(t, M) \in \operatorname{Gal}\left(K_{m^{-\infty}} / K\right) \mid M \in \mathcal{G}\left(m^{\infty}\right) \text { and } t \in \operatorname{Im}(M-I)\right\} .
$$

In view of (4.8), we consider the sets

$$
S_{x, \mathrm{~F}}:=\left\{(t, M) \in \operatorname{Gal}\left(K_{m^{-\infty} \alpha} / K\right) \mid M \in \mathcal{M}_{x, \mathrm{~F}} \text { and } t \in \operatorname{Im}(M-I)\right\} .
$$

By assertion (4) of Proposition 13 and our assumption that $(A / K, m, \alpha)$ satisfies eventual maximal growth of the torsion fields, the set $S_{\alpha}$ is the disjoint union of the sets $S_{x, \mathrm{~F}}$ up to a set of measure 0 . To see that the Haar measure of $S_{x, \mathrm{~F}}$ is well defined and to compute it, we define for every $n \geqslant 1$ the set

$$
S_{x, \mathrm{~F}, m^{n}}=\left\{(t, M) \in \operatorname{Gal}\left(K_{m^{-n} \alpha} / K\right) \mid M \in \mathcal{M}_{x, \mathrm{~F}}\left(m^{n}\right) \text { and } t \in \operatorname{Im}(M-I)\right\} .
$$

PROpOSITION 14. Suppose $n>n_{0}$ and $n>\max _{\ell}\left\{v_{\ell}(\exp \mathrm{F})\right\}$ for every $\ell$, where $n_{0}$ is as in Definition 3. Then the set $S_{x, \mathrm{~F}, m^{n}}$ is the image of $S_{x, \mathrm{~F}}$ under the projection to $\operatorname{Gal}\left(K_{m^{-n} \alpha} / K\right)$.

Proof. The set $S_{x, \mathrm{~F}, m^{n}}$ clearly contains the reduction modulo $m^{n}$ of $S_{x, \mathrm{~F}}$. To prove the other inclusion, consider $\left(t_{m^{n}}, M_{m^{n}}\right) \in S_{x, \mathrm{~F}, m^{n}}$ and a lift $(t, M) \in \operatorname{Gal}\left(K_{m^{-\infty} \alpha} / K\right)$. Since $n$ is sufficiently large with respect to $F$, we have $\operatorname{ker}(M-I) \cong F$. Clearly, $M_{m^{n}}$ and $M$ have the same projection $x \in \mathcal{G}(m)$. To conclude, it suffices to ensure $t \in \operatorname{Im}(M-I)$. Take $\tau_{m^{n}} \in A\left[m^{n}\right]$ satisfying $\left(M_{m^{n}}-I\right)\left(\tau_{m^{n}}\right)=t_{m^{n}}$, and some lift $\tau$ of $\tau_{m^{n}}$ to $T_{m}(A)$ : we may replace $t$ by $(M-I) \tau$ because the difference is in $m^{n} T_{m}(A)$ and since $n>n_{0}$ we know that $\operatorname{Gal}\left(K_{m^{-\infty}} / K\right)$ contains $m^{n} T_{m}(A) \times\{I\}$.

THEOREM 15. We have

$$
\mu\left(S_{x, \mathrm{~F}}\right)=\frac{\mathrm{C}_{m}}{\# \mathrm{~F}} \int_{\mathcal{M}_{x, \mathrm{~F}}} \mathrm{w}_{m^{\infty}}(M) d \mu_{\mathcal{G}\left(m^{\infty}\right)}(M),
$$

where $\mathrm{C}_{m}$ is the constant of (3.2) and $\mathrm{w}_{m} \infty$ is as in (4.6).

Proof. Choose $n$ large enough so that $n>n_{0}$ and $n>\max _{\ell}\left\{v_{\ell}(\exp \mathrm{F})\right\}$ for every $\ell$, where $n_{0}$ is as in Definition 3. By definition (see (4.1)) we can write

$$
\# S_{x, \mathrm{~F}, m^{n}}=\sum_{M \in \mathcal{M}_{x, \mathrm{~F}}\left(m^{n}\right)} \#\left(\operatorname{Im}(M-I) \cap \mathcal{W}_{m^{n}}(M)\right) .
$$

By definition (see (4.2)), we can express the summand as

$$
\# \operatorname{Im}(M-I) \cdot \mathrm{w}_{m^{n}}(M)=\frac{\mathrm{w}_{m^{n}}(M) \cdot m^{b n}}{\# \mathrm{~F}},
$$

so from (3.2), we deduce

$$
\frac{\# S_{x, \mathrm{~F}, m^{n}}}{\# \operatorname{Gal}\left(K_{m^{-n} \alpha} / K\right)}=\frac{1}{\# \mathcal{G}\left(m^{n}\right)} \sum_{M \in \mathcal{M}_{x, \mathrm{~F}}\left(m^{n}\right)} \frac{\mathrm{C}_{m}}{\# \mathrm{~F}} \cdot \mathrm{w}_{m^{n}}(M) .
$$

By (3.1), the left-hand side is a non-increasing function of $n$, and therefore, it admits a limit for $n \rightarrow \infty$, which is $\mu\left(S_{x, \mathrm{~F}}\right)$. The right-hand side is an integral over $\mathcal{M}_{x, \mathrm{~F}}\left(m^{n}\right)$ with 
respect to the normalised counting measure of $\mathcal{G}\left(\mathrm{m}^{n}\right)$, and the matrices in $\mathcal{M}_{x, \mathrm{~F}}$ are exactly the matrices in $\mathcal{G}\left(m^{\infty}\right)$ whose reduction modulo $m^{n}$ lies in $\mathcal{M}_{x, \mathrm{~F}}\left(m^{n}\right)$. Taking the limit over $n$, we thus find the formula in the statement.

THEOREM 16. We have

$$
\begin{aligned}
\operatorname{Dens}_{m}(\alpha) & =\mathrm{C}_{m} \sum_{\mathrm{F}} \frac{1}{\# \mathrm{~F}} \int_{\mathcal{M}_{\mathrm{F}}} \mathrm{w}_{m^{\infty}}(M) d \mu_{\mathcal{G}\left(m^{\infty}\right)}(M) \\
& =\mathrm{C}_{m} \int_{\mathcal{G}\left(m^{\infty}\right)} \frac{\mathrm{w}_{m^{\infty}}(M)}{\# \operatorname{ker}(M-I)} d \mu_{\mathcal{G}\left(m^{\infty}\right)}(M),
\end{aligned}
$$

where the function $\mathrm{w}_{m} \infty$ is as in (4.6), the constant $\mathrm{C}_{m}$ is as in (3.2), and $\mathrm{F}$ varies over the products over the primes $\ell \mid m$ of finite abelian $\ell$-groups with at most $b_{A}$ cyclic components.

Proof. To prove the first equality, note that $\mathcal{M}_{\mathrm{F}}$ is the disjoint union of the $\mathcal{M}_{x, \mathrm{~F}}$ for $x \in$ $\mathcal{G}(m)$. By Theorem 7, we may write Dens $m(\alpha)=\mu\left(S_{\alpha}\right)=\sum_{x, \mathrm{~F}} \mu\left(S_{x, \mathrm{~F}}\right)$ and then it suffices to apply Theorem 15 . The second equality follows because the union of the sets $\mathcal{M}_{\mathrm{F}}$ from (4.7) has measure 1 in $\mathcal{G}\left(m^{\infty}\right)$ by Proposition 13.

COROLlary 17 ([4, Theorem 1 and Remark 27]). In the special case $m=\ell$, we have

$$
\begin{aligned}
\operatorname{Dens}_{\ell}(\alpha) & =\mathrm{C}_{\ell} \sum_{\mathrm{F}} \frac{1}{\# \mathrm{~F}} \int_{\mathcal{M}_{\mathrm{F}}} \mathrm{w}_{\ell \infty}(M) d \mu_{\mathcal{G}\left(\ell^{\infty}\right)}(M) \\
& =\mathrm{C}_{\ell} \int_{\mathcal{G}\left(\ell^{\infty}\right)} \frac{\mathrm{w}_{\ell^{\infty}}(M)}{\# \operatorname{ker}(M-I)} d \mu_{\mathcal{G}\left(\ell^{\infty}\right)}(M),
\end{aligned}
$$

where $\mathrm{F}$ varies among the finite abelian $\ell$-groups with at most $b_{A}$ cyclic components.

Notice that we have $\# \operatorname{ker}(M-I)=\ell^{v_{\ell}(\operatorname{det}(M-I))}$ for every $M \in \mathcal{G}\left(\ell^{\infty}\right)$; this shows the equivalence with [4, Theorem 1$]$.

COROLlary 18. Let $\ell$ vary among the prime divisors of $m$. If the fields $K_{\ell^{-\infty}}$ are linearly disjoint over $K$, then we have

$$
\operatorname{Dens}_{m}(\alpha)=\prod_{\ell} \operatorname{Dens}_{\ell}(\alpha) .
$$

Proof. Note that we have $\mathrm{C}_{m}=\prod_{\ell} \mathrm{C}_{\ell}$. By assumption, we also have $\mathcal{G}\left(m^{\infty}\right)=$ $\prod_{\ell} \mathcal{G}\left(\ell^{\infty}\right)$, which implies $\mu_{\mathcal{G}\left(m^{\infty}\right)}=\prod_{\ell} \mu_{\mathcal{G}\left(\ell^{\infty}\right)}$, and $\mathrm{w}_{m^{\infty}}(M)=\prod_{\ell} \mathrm{w}_{\ell^{\infty}}\left(\pi_{\ell} M\right)$. We conclude that (5.1) is the product of the expressions (5.2) for $\ell \mid m$.

The conditions of Corollary 18 are satisfied, for example, if $K_{m^{-1}}=K$, or more generally if the degree $\left[K_{\ell^{-1}}: K\right]$ is a power of $\ell$ for each $\ell$. Under weaker conditions, $\operatorname{Dens}_{m}(\alpha)$ is not in general the product of the $\operatorname{Dens}_{\ell}(\alpha)$, but we can still express it as a sum of products of $\ell$-adic integrals, as the following result shows.

THEOREM 19. Denote by $H(x)=\prod_{\ell} H_{\ell}(x)$ the set of matrices in $\mathcal{G}\left(m^{\infty}\right) \subseteq \prod_{\ell} \mathcal{G}\left(\ell^{\infty}\right)$ mapping to $x$ in $\mathcal{G}(m)$. We then have

$$
\operatorname{Dens}_{m}(\alpha)=\frac{\mathrm{C}_{m}}{\# \mathcal{G}(m)} \sum_{x \in \mathcal{G}(m)} \prod_{\ell} \int_{H_{\ell}(x)} \frac{\mathrm{w}_{x, \ell^{\infty}}(M)}{\# \operatorname{ker}(M-I)} d \mu_{H_{\ell}(x)}(M),
$$

where $\mathrm{w}_{x, \ell^{\infty}}$ is as in (4.5). 
Proof. Write $S_{x}=\bigcup_{\mathrm{F}} S_{x, \mathrm{~F}}$ and recall from Proposition 13 that the set of matrices $M$ for which $\operatorname{ker}(M-I)$ is infinite has measure zero in $\mathcal{G}\left(m^{\infty}\right)$. By Theorem 15, we have

$$
\mu\left(S_{x}\right)=\sum_{\mathrm{F}} \mu\left(S_{x, \mathrm{~F}}\right)=\mathrm{C}_{m} \int_{H(x)} \frac{\mathrm{w}_{m^{\infty}}(M)}{\# \operatorname{ker}(M-I)} d \mu_{\mathcal{G}\left(m^{\infty}\right)}(M) .
$$

The assertion follows from Propositions 2 and 11.

COROllary 20. The density Dens ${ }_{m}(\alpha)$ is a rational number. Moreover, for every positive integer $b$, there exists a non-zero polynomial $p_{b}(t) \in \mathbb{Z}[t]$ with the following property: whenever $K$ is a number field and $A$ is the product of an abelian variety and a torus such that the first Betti number of $A$ equals $b$, then for all $\alpha \in A(K)$ and all square-free integers $m \geqslant 2$ such that $(A / K, m, \alpha)$ satisfies eventual maximal growth of the Kummer extensions, we have

$$
\operatorname{Dens}_{m}(\alpha) \cdot \prod_{\ell} p_{b}(\ell) \in \mathbb{Z}[1 / m],
$$

where $\ell$ varies over the prime divisors of $m$.

Proof. Recall that $\mathrm{C}_{m}$ is an integer. In view of Lemma 10, we can consider each $\ell$-adic integral in (5.3) and proceed as in the proof of [4, Theorem 36].

REMARK 21. For elliptic curves, it is also possible to bound the minimal denominator of Dens ${ }_{m}(\alpha)$. Indeed, let us consider (5.3), recalling that $\mathrm{C}_{m}$ is an integer. Each of the finitely many functions $\mathrm{w}_{x, \ell^{\infty}}$ takes only finitely many values: these are rational numbers whose minimal denominator divides $\ell^{2 n_{0}}$, where $n_{0}$ is large enough so that condition (3.1) holds for all $N \geqslant n \geqslant n_{0}$. If $M \in \mathcal{M}_{\ell}(a, b)$ (see Section 6.2), then $\# \operatorname{ker}(M-I)=\ell^{2 a+b}$. The crucial fact is the independence of the number of lifts [3, Theorem 28]; the case distinction for the normaliser of a Cartan subgroup does not matter because we separately count the matrices in the Cartan subgroup and those in its complement. This means that the measure of $\mathcal{M}_{\ell}(a, b) \cap H_{\ell}(x)$ is a fraction of that of $\mathcal{M}_{\ell}(a, b)$ : this ratio can take only finitely many values and can be understood by working modulo $\ell^{n_{0}}$. We may then need to multiply the denominator in the measure of $\mathcal{M}_{\ell}(a, b)$ by an integer which is at most $\# \mathrm{GL}_{2}\left(\ell^{n_{0}}\right)$. Essentially we need to evaluate finitely many geometric series because of the eventual maximal growth of the torsion fields (the degrees $\left[K\left(E\left[\ell^{n}\right]\right): K\right]$ for $n$ sufficiently large form a geometric progression) and we may reason as in [4, Theorems 5 and 6].

REMARK 22. We may replace the point $\alpha$ by a finitely generated subgroup $G$ of $A(K)$. Indeed, let $\alpha_{1}, \ldots, \alpha_{r}$ be generators for $G$. We may then consider the point $\beta=$ $\left(\alpha_{1}, \ldots, \alpha_{r}\right)$ in the product $A^{r}(K)$. Then the density $\operatorname{Dens}_{m}(\beta)$ for the single point $\beta$ is exactly the density of primes $\mathfrak{p}$ of $K$ such that the order of $(G \bmod \mathfrak{p})$ is coprime to $m$.

\section{Serre curves.}

6.1. Definition of Serre curves. Let $E$ be an elliptic curve over a number field $K$. We choose a Weierstrass equation for $E$ of the form

$$
E: y^{2}=\left(x-x_{1}\right)\left(x-x_{2}\right)\left(x-x_{3}\right),
$$

where $x_{1}, x_{2}, x_{3} \in K(E[2])$ are the $x$-coordinates of the points of order 2 . The discriminant of the right-hand side of (6.1) is $\Delta=\sqrt{\Delta}^{2}$, where 


$$
\sqrt{\Delta}=\left(x_{1}-x_{2}\right)\left(x_{2}-x_{3}\right)\left(x_{3}-x_{1}\right) .
$$

We thus have $K(\sqrt{\Delta}) \subseteq K(E[2])$, and we define a character

$$
\begin{aligned}
\psi_{E}: \operatorname{Gal}(K(E[2]) / K) & \longrightarrow\{ \pm 1\} \\
\sigma & \longmapsto \sigma(\sqrt{\Delta}) / \sqrt{\Delta} .
\end{aligned}
$$

For any choice of basis of the 2-torsion of $E$, we have the 2-torsion representation

$$
\rho_{E, 2}: \operatorname{Gal}(K(E[2]) / K) \longrightarrow \mathrm{GL}_{2}(\mathbb{Z} / 2 \mathbb{Z}) .
$$

Let $\psi$ be the unique non-trivial character $\mathrm{GL}_{2}(\mathbb{Z} / 2 \mathbb{Z}) \rightarrow\{ \pm 1\}$; this corresponds to the sign character under any isomorphism of $\mathrm{GL}_{2}(\mathbb{Z} / 2 \mathbb{Z})$ with $S_{3}$. The character $\psi_{E}$ factors as

$$
\psi_{E}=\psi \circ \rho_{E, 2} \text {. }
$$

From now on, we take $K=\mathbb{Q}$. All number fields that we will consider will be subfields of a fixed algebraic closure $\overline{\mathbb{Q}}$ of $\mathbb{Q}$.

Let $d$ be an element of $\mathbb{Q}^{\times}$. Let $m_{d}$ be the conductor of $\mathbb{Q}(\sqrt{d})$; this is the smallest positive integer such that $\sqrt{d}$ lies in the cyclotomic field $\mathbb{Q}\left(\zeta_{m_{d}}\right)$. Let $d_{\mathrm{sf}}$ be the square-free part of $d$. We have

$$
m_{d}= \begin{cases}\left|d_{\mathrm{sf}}\right| & \text { if } d_{\mathrm{sf}} \equiv 1 \bmod 4 \\ 4\left|d_{\mathrm{sf}}\right| & \text { otherwise }\end{cases}
$$

We define a character

$$
\begin{aligned}
\varepsilon_{d}: \operatorname{Gal}\left(\mathbb{Q}\left(\zeta_{m_{d}}\right) / \mathbb{Q}\right) & \longrightarrow\{ \pm 1\} \\
\sigma & \longmapsto \sigma(\sqrt{d}) / \sqrt{d} .
\end{aligned}
$$

If $\sigma$ is the automorphism of $\mathbb{Q}\left(\zeta_{m_{d}}\right)$ defined by $\sigma\left(\zeta_{m_{d}}\right)=\zeta_{m_{d}}^{a}$ with $a \in\left(\mathbb{Z} / m_{d} \mathbb{Z}\right)^{\times}$, then $\varepsilon_{d}(\sigma)$ equals the Jacobi symbol $\left(\frac{d_{\mathrm{sf}}}{a}\right)$. We view $\varepsilon_{d}$ as a character of $\mathrm{GL}_{2}\left(\mathbb{Z} / m_{d} \mathbb{Z}\right)$ by composing with the determinant.

For all $n \geqslant 1$, we have a canonical projection

$$
\pi_{n}: \mathrm{GL}_{2}(\widehat{\mathbb{Z}}) \rightarrow \mathrm{GL}_{2}(\mathbb{Z} / n \mathbb{Z}) .
$$

Fixing a $\widehat{\mathbb{Z}}$-basis for the projective limit of the torsion groups $E[n](\overline{\mathbb{Q}})$, we have a torsion representation

$$
\rho_{E}: \operatorname{Gal}(\overline{\mathbb{Q}} / \mathbb{Q}) \rightarrow \mathrm{GL}_{2}(\widehat{\mathbb{Z}}) .
$$

The image of $\rho_{E}$ is contained in the subgroup

$$
H_{\Delta}=\left\{M \in \mathrm{GL}_{2}(\widehat{\mathbb{Z}}) \mid \psi\left(\pi_{2}(M)\right)=\varepsilon_{\Delta}\left(\pi_{m_{\Delta}}(M)\right)\right\}
$$

of index 2 in $\mathrm{GL}_{2}(\widehat{\mathbb{Z}})$. This expresses the fact that $\sqrt{\Delta}$ is contained in both $\mathbb{Q}(E[2])$ and $\mathbb{Q}\left(E\left[m_{\Delta}\right]\right)$. An elliptic curve is said to be a Serre curve if the image of $\rho_{E}$ is equal to $H_{\Delta}$. As proven by N. Jones [1], almost all elliptic curves over $\mathbb{Q}$ are Serre curves.

6.2. Counting matrices. Let $\ell$ be a prime number. For all integers $a, b \geqslant 0$, we write $\mathcal{M}_{\ell}(a, b)$ for the set of matrices $M \in \mathrm{GL}_{2}\left(\mathbb{Z}_{\ell}\right)$ such that the kernel of $M-I$ as an endomorphism of $\left(\mathbb{Q}_{\ell} / \mathbb{Z}_{\ell}\right)^{2}$ is isomorphic to $\mathbb{Z} / \ell^{a} \mathbb{Z} \times \mathbb{Z} / \ell^{a+b} \mathbb{Z}$. 
If $\mathcal{N}$ is a non-empty subset of $\mathcal{M}_{\ell}(a, b)$ that is the preimage in $\mathcal{M}_{\ell}(a, b)$ of its reduction modulo $\ell^{n}$ (which means that $\mathcal{N}$ contains the intersection of $\mathcal{M}_{\ell}(a, b)$ with the set of preimages of $\left(\mathcal{N} \bmod \ell^{n}\right)$ in $\left.\mathrm{GL}_{2}\left(\mathbb{Z}_{\ell}\right)\right)$, then we have

$$
\frac{\mu_{\mathrm{GL}_{2}\left(\mathbb{Z}_{\ell}\right)}(\mathcal{N})}{\mu_{\mathrm{GL}_{2}\left(\mathbb{Z}_{\ell}\right)}\left(\mathcal{M}_{\ell}(a, b)\right)}=\frac{\mu_{\mathrm{GL}_{2}\left(\mathbb{Z} / \ell^{n} \mathbb{Z}\right)}\left(\mathcal{N} \bmod \ell^{n}\right)}{\mu_{\mathrm{GL}_{2}\left(\mathbb{Z} / \ell^{n} \mathbb{Z}\right)}\left(\mathcal{M}_{\ell}(a, b) \bmod \ell^{n}\right)}
$$

by [3, Theorem 27] (where the number of lifts is independent of the matrix). Notice that if $a \geqslant n$, then $\left(\mathcal{N} \bmod \ell^{n}\right)$ consists of the identity.

Proposition 23. If $\mathcal{N}$ is a subset of $\mathcal{M}_{\ell}(a, b)$ that is the preimage in $\mathcal{M}_{\ell}(a, b)$ of its reduction modulo $\ell$, then we have

$$
\mu_{\mathrm{GL}_{2}\left(\mathbb{Z}_{\ell}\right)}(\mathcal{N})=\mu_{\mathrm{GL}_{2}(\mathbb{Z} / \ell \mathbb{Z})}(\mathcal{N} \bmod \ell) \cdot \begin{cases}1 & \text { if } a=b=0 \\ \ell^{-b}(\ell-1) & \text { if } a=0, b \geqslant 1 \\ \ell^{-4 a} \cdot \ell(\ell-1)^{2}(\ell+1) & \text { if } a \geqslant 1, b=0 \\ \ell^{-4 a-b} \cdot(\ell-1)^{2}(\ell+1)^{2} & \text { if } a \geqslant 1, b \geqslant 1 .\end{cases}
$$

Proof. We are working with $\mathrm{GL}_{2}\left(\mathbb{Z}_{\ell}\right)$, so we can apply [3, Proposition 33] (see also [3, Definition 19]). This gives the assertion for the set $\mathcal{M}_{\ell}(a, b)$; we can conclude because of (6.2).

We now collect some results in the case $\ell=2$. From [3, Theorem 2], we know

$$
\mu_{\mathrm{GL}_{2}\left(\mathbb{Z}_{2}\right)}\left(\mathcal{M}_{2}(a, b)\right)= \begin{cases}1 / 3 & \text { if } a=b=0 \\ 1 / 2 \cdot 2^{-b} & \text { if } a=0, b \geqslant 1 \\ 2^{-4 a} & \text { if } a \geqslant 1, b=0 \\ 3 / 2 \cdot 2^{-4 a-b} & \text { if } a \geqslant 1, b \geqslant 1 .\end{cases}
$$

We consider the action of $\mathrm{GL}_{2}\left(\mathbb{Z} / 2^{3} \mathbb{Z}\right)$ on $\mathbb{Q}\left(\zeta_{2^{3}}\right)$ defined by $M \zeta_{2^{3}}=\zeta_{2^{3}}^{\operatorname{det} M}$. The matrices $M \in \mathrm{GL}_{2}\left(\mathbb{Z} / 2^{3} \mathbb{Z}\right)$ that fix $\sqrt{-1}$ are those with $\operatorname{det}(M)=1,5$. The ones that fix $\sqrt{2}$ are those with $\operatorname{det}(M)=1,7$. The ones that fix $\sqrt{-2}$ are those with $\operatorname{det}(M)=1,3$.

For $a, b \in\{0,1,2,3\}$ and $z \in\{-1,2,-2\}$, we write $\mathcal{N}_{2}(a, b, z)$ for the set of matrices in $\mathcal{M}_{2}(a, b)$ that fix $\sqrt{z}$.

LEMMA 24. We have

$$
\frac{\mu_{\mathrm{GL}_{2}\left(\mathbb{Z}_{2}\right)}\left(\mathcal{N}_{2}(a, b ;-1)\right)}{\mu_{\mathrm{GL}_{2}\left(\mathbb{Z}_{2}\right)}\left(\mathcal{M}_{2}(a, b)\right)}= \begin{cases}1 / 2 & \text { for } a=0, b \geqslant 0 \\ 2 / 3 & \text { for } a=1, b=0 \\ 1 / 3 & \text { for } a=1, b \geqslant 1 \\ 1 & \text { for } a \geqslant 2, b \geqslant 0\end{cases}
$$


and

$$
\frac{\mu_{\mathrm{GL}_{2}\left(\mathbb{Z}_{2}\right)}\left(\mathcal{N}_{2}(a, b ; \pm 2)\right)}{\mu_{\mathrm{GL}_{2}\left(\mathbb{Z}_{2}\right)}\left(\mathcal{M}_{2}(a, b)\right)}= \begin{cases}1 / 2 & \text { for } a \leqslant 1, b \geqslant 0 \\ 2 / 3 & \text { for } a=2, b=0 \\ 1 / 3 & \text { for } a=2, b \geqslant 1 \\ 1 & \text { for } a \geqslant 3, b \geqslant 0 .\end{cases}
$$

Proof. For $a, b \in\{0,1,2,3\}$ and $d \in\left(\mathbb{Z} / 2^{3} \mathbb{Z}\right)^{\times}$, let $h(a, b, d)$ be the number of matrices $M \in \mathrm{GL}_{2}\left(\mathbb{Z} / 2^{3} \mathbb{Z}\right)$ such that $\operatorname{det}(M)=d$ and $\operatorname{ker}(M-I) \cong \mathbb{Z} / 2^{a} \mathbb{Z} \times \mathbb{Z} / 2^{a+b} \mathbb{Z}$. Using [9] one can easily count these matrices:

- $h(0,0, d)=128, h(0,1, d)=96$ and $h(0,2, d)=h(0,3, d)=48$ for all $d$;

- $h(1,0, d)=32$ for $d=1,5$ and $h(1,0, d)=16$ for $d=3,7$;

- for $b=1,2$ we have $h(1, b, d)=12$ for $d=1,5$ and $h(1, b, d)=24$ for $d=3,7$;

- $h(2,0,1)=4, h(2,0,5)=2$ and $h(2,0, d)=0$ for $d=3,7$;

- $h(2,1,1)=3, h(2,1,5)=6$ and $h(2,1, d)=0$ for $d=3,7$;

- $h(3,0,1)=1$ (the identity matrix) and $h(3,0, d)=0$ for $d=3,5,7$.

This classification and (6.2) lead to the measures in the statement.

LEMMA 25. For all $a, b \geq 0$ and all $M \in \mathcal{M}_{2}(a, b)$, we have

$$
\psi(M)= \begin{cases}-1 & \text { if } a=0 \text { and } b \geqslant 1, \\ 1 & \text { otherwise. }\end{cases}
$$

Proof. Consider matrices $M \in \mathrm{GL}_{2}(\mathbb{Z} / 2 \mathbb{Z})$. The matrices

$$
M \in\left\{\left(\begin{array}{ll}
1 & 0 \\
0 & 1
\end{array}\right), \quad\left(\begin{array}{ll}
0 & 1 \\
1 & 1
\end{array}\right), \quad\left(\begin{array}{ll}
1 & 1 \\
1 & 0
\end{array}\right)\right\}
$$

satisfy $\psi(M)=1$ and $\operatorname{dim}_{\mathbb{F}_{2}} \operatorname{ker}(M-I) \in\{0,2\}$. The matrices

$$
M \in\left\{\left(\begin{array}{ll}
0 & 1 \\
1 & 0
\end{array}\right), \quad\left(\begin{array}{ll}
1 & 1 \\
0 & 1
\end{array}\right), \quad\left(\begin{array}{ll}
1 & 0 \\
1 & 1
\end{array}\right)\right\}
$$

satisfy $\psi(M)=-1$ and $\operatorname{dim}_{\mathbb{F}_{2}} \operatorname{ker}(M-I)=1$. This implies the claim.

Now let $\ell$ be an odd prime number. We write

$$
\ell^{*}=(-1)^{(\ell-1) / 2} \ell,
$$

so $\varepsilon_{\ell^{*}}$ is a character of $(\mathbb{Z} / \ell \mathbb{Z})^{\times}$and also of $\mathrm{GL}_{2}(\mathbb{Z} / \ell \mathbb{Z})$ via the determinant.

LEMMA 26. Let $M$ vary in $\mathrm{GL}_{2}(\mathbb{Z} / \ell \mathbb{Z}) \backslash\{I\}$, where $\ell$ is an odd prime number.

(1) There are $\frac{1}{2}(\ell+1)^{2}(\ell-2)$ matrices $M$ satisfying $\varepsilon_{\ell^{*}}(M)=1$ and $\ell \mid \operatorname{det}(M-I)$.

(2) There are $\frac{1}{2} \ell\left(\ell^{3}-2 \ell^{2}-\ell+4\right)$ matrices $M$ satisfying $\varepsilon_{\ell^{*}}(M)=1$ and $\ell \nmid$ $\operatorname{det}(M-I)$.

(3) There are $\frac{1}{2} \ell\left(\ell^{2}-1\right)$ matrices $M$ satisfying $\varepsilon_{\ell^{*}}(M)=-1$ and $\ell \mid \operatorname{det}(M-I)$.

(4) There are $\frac{1}{2} \ell\left(\ell^{2}-1\right)(\ell-2)$ matrices $M$ satisfying $\varepsilon_{\ell^{*}}(M)=-1$ and $\ell \nmid$ $\operatorname{det}(M-I)$. 
Proof. (1) Write $\chi(M)$ for the characteristic polynomial of $M$. The condition $\varepsilon_{\ell^{*}}(M)=1$ is equivalent to $\operatorname{det}(M)=\chi(0)$ being a square in $(\mathbb{Z} / \ell \mathbb{Z})^{\times}$, and the condition $\ell \mid \operatorname{det}(M-I)$ is equivalent to $\chi(1)=0$ in $\mathbb{Z} / \ell \mathbb{Z}$. Thus, the matrices $M$ satisfying both conditions are those for which there exists $s \in(\mathbb{Z} / \ell \mathbb{Z})^{\times}$with

$$
\chi(M)=(x-1)\left(x-s^{2}\right) .
$$

The matrices with $\chi(0) \neq 1$ (giving $\frac{\ell-1}{2}-1$ possibilities for $\chi$ ) are diagonalisable, and we only have to choose the two distinct eigenspaces; this gives $(\ell+1) \ell$ matrices for every such $\chi$. The matrices with $\chi(0)=1$ are the identity (which we are excluding) and the $\ell^{2}-1$ matrices conjugate to $\left(\begin{array}{ll}1 & 1 \\ 0 & 1\end{array}\right)$. Note that (1) can also be obtained from [7, Table 1].

(2) There are $\frac{1}{2} \# \mathrm{GL}_{2}(\mathbb{Z} / \ell \mathbb{Z})$ matrices satisfying $\varepsilon_{\ell^{*}}=1$, and we only need to subtract the identity and the matrices from (1).

(3) There are $\ell^{3}-2 \ell$ matrices in $\mathrm{GL}_{2}(\mathbb{Z} / \ell \mathbb{Z})$ having 1 as an eigenvalue (see for example [3, Proof of Theorem 2]), and we only need to subtract the identity and the matrices from (1).

(4) There are $\frac{1}{2} \# \mathrm{GL}_{2}(\mathbb{Z} / \ell \mathbb{Z})$ matrices satisfying $\varepsilon_{\ell^{*}}=-1$, and we only need to subtract the matrices from (3). Alternatively, there are $\# \mathrm{GL}_{2}(\mathbb{Z} / \ell \mathbb{Z})-\left(\ell^{3}-2 \ell\right)$ matrices that do not have 1 as eigenvalue, and we only need to subtract the matrices from (2).

6.3. Partitioning the image of the $\boldsymbol{m}$-adic representation. Let $E$ be a Serre curve over $\mathbb{Q}$. Let $\Delta$ be the minimal discriminant of $E$, and let $\Delta_{\text {sf }}$ be its square-free part. We write $\Delta_{\text {sf }}=z u$, where $z \in\{1,-1,2,-2\}$ and where $u$ is an odd fundamental discriminant. Then $|u|$ is the odd part of $m_{\Delta}$, and we have $\varepsilon_{\Delta}=\varepsilon_{z} \cdot \varepsilon_{u}$ as characters of $\left(\mathbb{Z} / m_{\Delta} \mathbb{Z}\right)^{\times}$.

Now let $m$ be a square-free positive integer. If $m=2$, or if $m$ is odd, or if $u$ does not divide $m$, then we have

$$
\mathcal{G}\left(m^{\infty}\right)=\prod_{\ell} \mathcal{G}\left(\ell^{\infty}\right) .
$$

If $m \neq 2$ is even and $u$ divides $m$, then $\mathcal{G}\left(m^{\infty}\right)$ has index 2 in $\prod_{\ell} \mathcal{G}\left(\ell^{\infty}\right)$. The defining condition for the image of the $m$-adic representation is then $\psi=\varepsilon_{\Delta}$, or equivalently

$$
\psi \cdot \varepsilon_{z}=\varepsilon_{u}
$$

We may then partition $\mathcal{G}\left(m^{\infty}\right) \subseteq \prod_{\ell \mid m} \mathcal{G}\left(\ell^{\infty}\right)$ into two sets that are products, namely

$$
\left(\mathcal{G}\left(2^{\infty}\right) \cap\left\{\psi \cdot \varepsilon_{z}=1\right\}\right) \times\left(\mathcal{G}\left(|u|^{\infty}\right) \cap\left\{\varepsilon_{u}=1\right\}\right) \times \mathcal{G}\left(\left|\frac{m}{2 u}\right|^{\infty}\right)
$$

and

$$
\left(\mathcal{G}\left(2^{\infty}\right) \cap\left\{\psi \cdot \varepsilon_{z}=-1\right\}\right) \times\left(\mathcal{G}\left(|u|^{\infty}\right) \cap\left\{\varepsilon_{u}=-1\right\}\right) \times \mathcal{G}\left(\left|\frac{m}{2 u}\right|^{\infty}\right) .
$$

The set $\mathcal{G}\left(|u|^{\infty}\right) \cap\left\{\varepsilon_{u}=1\right\}$ is the disjoint union of sets of the form $\prod_{\ell \mid u}\left(\mathcal{G}\left(\ell^{\infty}\right) \cap\left\{\varepsilon_{\ell^{*}}=\right.\right.$ $\pm 1\}$ ), choosing an even number of minus signs; for the set $\mathcal{G}\left(|u|^{\infty}\right) \cap\left\{\varepsilon_{u}=-1\right\}$ we have to choose an odd number of minus signs. Since each $\ell \mid u$ is odd, the two sets $\mathcal{G}\left(\ell^{\infty}\right) \cap\left\{\varepsilon_{\ell^{*}}=\right.$ $\pm 1\}$ can be investigated with the help of Lemma 26. Finally, the two sets $\mathcal{G}\left(2^{\infty}\right) \cap\left\{\psi \cdot \varepsilon_{z}=\right.$ $\pm 1\}$ can be investigated using Lemmas 24 and 25 . 


\section{Examples.}

7.1. Example (non-surjective mod 3 representation). Consider the non-CM elliptic curve

$$
E: y^{2}+y=x^{3}+6 x+27
$$

of discriminant $-3^{19} \cdot 17$ and conductor $153=3^{2} \cdot 17$ over $\mathbb{Q}[8$, label 153.b2]. The group $E(\mathbb{Q})$ is infinite cyclic and is generated by the point

$$
\alpha=(5,13) \text {. }
$$

We will compute the following values (by testing the primes up to $10^{6}$, we have computed an approximation to $\operatorname{Dens}_{6}(\alpha)$ using [9]):

\begin{tabular}{|l|rl|r|c|}
\hline Point & \multicolumn{1}{|c|}{ Dens $_{2}$} & Dens $_{3}$ & \multicolumn{1}{|c|}{ Dens $_{6}$} & primes $<10^{6}$ \\
\hline$\alpha=(5,13)$ & $11 / 21$ & $23 / 104$ & $253 / 2184=11.584 \ldots \%$ & $11.624 \%$ \\
$2 \alpha=(-1,4)$ & $16 / 21$ & $23 / 104$ & $46 / 273=16.849 \ldots \%$ & $16.885 \%$ \\
$3 \alpha=(-7 / 4,-31 / 8)$ & $11 / 21$ & $77 / 104$ & $121 / 312=38.782 \ldots \%$ & $38.730 \%$ \\
$6 \alpha=(137 / 16,1669 / 64)$ & $16 / 21$ & $77 / 104$ & $22 / 39=56.410 \ldots \%$ & $56.373 \%$ \\
$4 \alpha=(3,-9)$ & $37 / 42$ & $23 / 104$ & $851 / 4368=19.482 \ldots \%$ & $19.479 \%$ \\
$9 \alpha=\left(\frac{19649}{12100},-\frac{9216643}{1331000}\right)$ & $11 / 21$ & $95 / 104$ & $1045 / 2184=47.847 \ldots \%$ & $47.791 \%$ \\
\hline
\end{tabular}

The image of the 3-adic representation is the inverse image of its reduction modulo 3 , the image of the mod 3 representation is isomorphic to the symmetric group of order 6 and the 3-adic Kummer map is surjective [4, Example 6.4]. The image of the mod 3 representation has a unique subgroup of index 2 , so the field $\mathbb{Q}(E[3])$ contains as its only quadratic subextension the cyclotomic field $\mathbb{Q}(\sqrt{-3})$.

The image of the 2-adic representation is $\mathrm{GL}_{2}\left(\mathbb{Z}_{2}\right)$; see [8]. By [2, Theorem 5.2], the 2-adic Kummer map is surjective: the assumptions of that result are satisfied because the prime $p=941$ splits completely in $E[4]$, but the point $(\alpha \bmod p)$ is not 2-divisible over $\mathbb{F}_{p}$. Since the image of the mod 2 representation has a unique subgroup of index 2 , the field $\mathbb{Q}(E[2])$ contains as its only quadratic subextension the field $\mathbb{Q}(\sqrt{-51})$ (the square-free part of the discriminant of $E$ is -51$)$.

We have $\mathbb{Q}(E[2]) \cap \mathbb{Q}(E[9])=\mathbb{Q}$ because the residual degree modulo 22699 of the extension $\mathbb{Q}(E[2], E[9]) / \mathbb{Q}(E[9])$ is divisible by 3 and the degree of this extension is even because $\mathbb{Q}(\sqrt{-51})$ is not contained in $\mathbb{Q}(E[3])$. We deduce $\mathbb{Q}(E[2]) \cap \mathbb{Q}\left(E\left[3^{\infty}\right]\right)=\mathbb{Q}$ by applying [4, Theorem 14 (i)] (where $K=\mathbb{Q}(E[2])$ ).

Moreover, we have $\mathbb{Q}(E[3]) \cap \mathbb{Q}(E[4])=\mathbb{Q}$ because $\mathbb{Q}(\sqrt{-3})$ is not contained in $\mathbb{Q}(E[4])$ : the prime 941 is not congruent to 1 modulo 3 and splits completely in $\mathbb{Q}(E[4])$. By $\left[4\right.$, Theorem 14 (i)], we conclude that $\mathbb{Q}(E[3]) \cap \mathbb{Q}\left(E\left[2^{\infty}\right]\right)=\mathbb{Q}$.

The 2-adic Kummer extensions of $\alpha$ have maximal degree also over $\mathbb{Q}(E[3])$, in view of the maximality of the 2-Kummer extension, because the prime 4349 splits completely in $\mathbb{Q}\left(2^{-2} \alpha\right)$ but not in $\mathbb{Q}(\sqrt{-3})$; see $[4$, Theorem 14 (ii)] (where $K=\mathbb{Q}(\sqrt{-3})$ ).

The 3-adic Kummer extensions of $\alpha$ have maximal degree also over $\mathbb{Q}(E[2])$ because the prime 217981 splits completely in $\mathbb{Q}\left(3^{-2} \alpha\right)$ but 3 divides the residual degree of $\mathbb{Q}(E[2])$; see $[4$, Theorem 14 (ii)] (where $K=\mathbb{Q}(E[2])$ ). 
We thus have $\mathcal{G}\left(6^{\infty}\right)=\mathcal{G}\left(2^{\infty}\right) \times \mathcal{G}\left(3^{\infty}\right)$, the $2^{\infty}$ Kummer extensions are independent from $\mathbb{Q}(E[3])$, and the $3^{\infty}$ Kummer extensions are independent from $\mathbb{Q}(E[2])$. We are thus in the situation that the fields $\mathbb{Q}\left(2^{-\infty} \alpha\right)$ and $\mathbb{Q}\left(3^{-\infty} \alpha\right)$ are linearly disjoint over $\mathbb{Q}$. We deduce from Corollary 18 that the equality

$$
\operatorname{Dens}_{6}(\alpha)=\operatorname{Dens}_{2}(\alpha) \cdot \operatorname{Dens}_{3}(\alpha)
$$

holds for $\alpha$ and for its multiples. The 2-densities can be evaluated by [4, Theorem 35], for the 3-densities see [4, Example 6.4].

7.2. The Serre curve $y^{2}+y=x^{3}+x^{2}$. The elliptic curve

$$
E: y^{2}+y=x^{3}+x^{2}
$$

of discriminant -43 and conductor 43 over $\mathbb{Q}$ [8, label 43.a1] is a Serre curve [6, Example 5.5.7]. The group $E(\mathbb{Q})$ is infinite cyclic and is generated by the point

$$
\alpha=(0,0) .
$$

The point $\alpha$ satisfies

$$
\operatorname{Dens}_{2}(\alpha) \cdot \operatorname{Dens}_{43}(\alpha) \neq \operatorname{Dens}_{2.43}(\alpha)
$$

because, as we will show below, we have

$$
\begin{gathered}
\operatorname{Dens}_{2}(\alpha)=\frac{11}{21}, \quad \operatorname{Dens}_{43}(\alpha)=\frac{143510179}{146927088}, \\
\operatorname{Dens}_{2}(\alpha) \cdot \operatorname{Dens}_{43}(\alpha)=\frac{143510179}{280497168} \sim 51.16279 \%, \\
\operatorname{Dens}_{2.43}(\alpha)=\frac{526206455}{1028489616} \sim 51.16303 \% .
\end{gathered}
$$

We will also compute the following values (by testing the primes up to $10^{6}$, we have computed an approximation to $\operatorname{Dens}_{2 \cdot 43}(\alpha)$ using [9]):

\begin{tabular}{|l|r|l|}
\hline Point & \multicolumn{1}{|c|}{ Dens $_{2.43}$} & primes $<10^{6}$ \\
\hline$\alpha=(0,0)$ & $526206455 / 1028489616=51.163 \ldots \%$ & $51.136 \%$ \\
$2 \alpha=(-1,-1)$ & $42521603 / 57138312=74.418 \ldots \%$ & $74.397 \%$ \\
$4 \alpha=(2,3)$ & $1769960107 / 2056979232=86.046 \ldots \%$ & $86.072 \%$ \\
\hline
\end{tabular}

By looking at the reduction modulo 293, we see that $\alpha$ is not divisible by 2 over the 4-torsion field of $E$. Therefore, by [2, Theorem 5.2], for every prime number $\ell$ and for every $n \geqslant 1$, the degree of the $\ell^{n}$-Kummer extension is maximal, i.e.

$$
\left[\mathbb{Q}_{\ell^{-n} \alpha}: \mathbb{Q}_{\ell^{-n}}\right]=\ell^{2 n} \text {. }
$$

The 43-adic Kummer extensions have maximal degree also over $\mathbb{Q}(E[2])$, i.e.

$$
\left[\mathbb{Q}_{43^{-n} \alpha}(E[2]): \mathbb{Q}_{43^{-n}}(E[2])\right]=43^{2 n},
$$

because the degree $[\mathbb{Q}(E[2]): \mathbb{Q}]=6$ is coprime to 43 . 
The extensions $\mathbb{Q}\left(2^{-1} \alpha\right)$ and $\mathbb{Q}(E[2 \cdot 43])$ are linearly disjoint over $\mathbb{Q}(E[2])$, as can be seen by investigating the residual degree for the reduction modulo the prime 29327 , which splits completely in $\mathbb{Q}(E[2])$. Indeed, the residual degree of the extension $\mathbb{Q}\left(2^{-1} \alpha\right)$ equals 4 , while the residual degree of the extension $\mathbb{Q}(E[2 \cdot 43])$ is odd because the prime is congruent to 1 modulo 43 , and there are points of order 43 in the reductions (the subgroup of the upper unitriangular matrices in $\mathrm{GL}_{2}(\mathbb{Z} / 43 \mathbb{Z})$ has order 43$)$.

The 2-adic Kummer extensions have maximal degree also over $\mathbb{Q}(E[43])$, i.e.

$$
\left[\mathbb{Q}_{2^{-n} \alpha}(E[43]): \mathbb{Q}_{2^{-n}}(E[43])\right]=2^{2 n} .
$$

To see this, we consider the intersection $L$ of $\mathbb{Q}_{2^{-n} \alpha}$ and $\mathbb{Q}(E[43])$. This is a Galois extension of $\mathbb{Q}$, and the group $G=\operatorname{Gal}(L / \mathbb{Q})$ is a quotient of both $\left(\mathbb{Z} / 2^{n} \mathbb{Z}\right)^{2} \rtimes \mathrm{GL}_{2}\left(\mathbb{Z} / 2^{n} \mathbb{Z}\right)$ and $\mathrm{GL}_{2}(\mathbb{Z} / 43 \mathbb{Z})$. Because $\mathrm{SL}_{2}(\mathbb{Z} / 43 \mathbb{Z})$ has no non-trivial quotient that can be embedded into a quotient of $\left(\mathbb{Z} / 2^{n} \mathbb{Z}\right)^{2} \rtimes \mathrm{GL}_{2}\left(\mathbb{Z} / 2^{n} \mathbb{Z}\right)$, the quotient map $\mathrm{GL}_{2}(\mathbb{Z} / 43 \mathbb{Z}) \rightarrow G$ factors as

$$
\mathrm{GL}_{2}(\mathbb{Z} / 43 \mathbb{Z}) \stackrel{\text { det }}{\longrightarrow}(\mathbb{Z} / 43 \mathbb{Z})^{\times} \longrightarrow G
$$

This implies that $L$ is a subfield of $\mathbb{Q}\left(\zeta_{43}\right)$. Furthermore, $L$ contains $\mathbb{Q}(\sqrt{-43})$. Because $\left(\mathbb{Z} / 2^{n} \mathbb{Z}\right)^{2} \rtimes \mathrm{GL}_{2}\left(\mathbb{Z} / 2^{n} \mathbb{Z}\right)$ does not have any quotient group of odd order, the maximal subfield of $\mathbb{Q}\left(\zeta_{43}\right)$ that can be embedded into $\mathbb{Q}_{2^{-n} \alpha}$ is $\mathbb{Q}(\sqrt{-43})$, and we conclude that $L$ equals $\mathbb{Q}(\sqrt{-43})$.

It follows that for $m=2 \cdot 43$, we have the maximal degree $\left[\mathbb{Q}_{m^{-n} \alpha}: \mathbb{Q}_{m^{-n}}\right]=m^{2 n}$ and, more generally, that for every multiple $P$ of $\alpha$ we have $\left[\mathbb{Q}_{m^{-n} P}: \mathbb{Q}_{m^{-n}}\right]=\left[\mathbb{Q}_{2^{-n} P}: \mathbb{Q}_{2^{-n}}\right]$. $\left[\mathbb{Q}_{43^{-n} P}: \mathbb{Q}_{43^{-n}}\right]$. We may then apply [4, Example 28] and various results in this paper to compute the exact densities in the above table, and we use [9] to numerically verify them for the primes up to $10^{6}$.

We conclude by sketching the computations for the point $\alpha$. The 43-adic representation is surjective, and the 43-Kummer extensions have maximal degree. By parts (3) and (4) of Lemma 26, we find that $\frac{1}{2 \cdot 42}$ (respectively, $\frac{41}{2 \cdot 42}$ ) is the counting measure in $\mathrm{GL}_{2}(\mathbb{Z} / 43 \mathbb{Z})$ of the matrices such that $\varepsilon_{-43}=-1$ and that are in $\left(\mathcal{M}_{43}(0, b) \bmod \ell\right)$ for some $b>0$ (respectively, for $b=0$ ). By multiplying this quantity by $43^{-b} \cdot 42$, we obtain by Proposition 23 that $\mu_{\mathrm{GL}_{2}\left(\mathbb{Z}_{43}\right)}\left(\mathcal{M}_{43}(0, b)\right)=\frac{1}{2} 43^{-b}$ for $b>0$. By [4, Example 28], the contribution to Dens 43 coming from the matrices in $\mathcal{G}\left(43^{\infty}\right)$ such that $\varepsilon_{-43}=-1$ is then

$$
\operatorname{Dens}_{43}\left(\varepsilon_{-43}=-1\right)=\frac{41}{2 \cdot 42}+\sum_{b>0} \frac{1}{2} \cdot 43^{-2 b}=\frac{1805}{2 \cdot 42 \cdot 44} .
$$

From [4, Theorem 35] we know that $\operatorname{Dens}_{43}(\alpha)=143510179 / 146927088$, and hence the contribution to $\operatorname{Dens}_{43}(\alpha)$ coming from the matrices in $\mathcal{G}\left(43^{\infty}\right)$ such that $\varepsilon_{-43}=+1$ equals

$$
\operatorname{Dens}_{43}\left(\varepsilon_{-43}=1\right)=\frac{3261637}{6678504} \text {. }
$$

Now we work with the 2-adic representation, which is surjective and restrict to counting the contribution to $\operatorname{Dens}_{2}(\alpha)$ coming from the matrices satisfying $\psi=-1$. In view of Lemma 25 and Proposition 23, we find $\mu_{\mathrm{GL}_{2}\left(\mathbb{Z}_{2}\right)}\left(\mathcal{M}_{2}(0, b)\right)=1 / 2 \cdot 2^{-b}$ for $b>0$. By [4, Example 28], the contribution to $\operatorname{Dens}_{2}(\alpha)$ coming from the matrices in $\mathcal{G}\left(2^{\infty}\right)$ such that $\psi=-1$ is therefore

$$
\operatorname{Dens}_{2}(\psi=-1)=\sum_{b>0} 1 / 2 \cdot 2^{-2 b}=1 / 6 .
$$


From [4, Theorem 35] we know that $\operatorname{Dens}_{2}(\alpha)=11 / 21$, and hence the contribution to Dens $_{2}$ coming from the matrices in $\mathcal{G}\left(2^{\infty}\right)$ such that $\psi=1$ is

$$
\operatorname{Dens}_{2}(\psi=1)=5 / 14 \text {. }
$$

Finally, by the partition in Section 6.3, we can compute the requested density as the following combination of the above quantities:

$$
\begin{aligned}
\operatorname{Dens}_{2 \cdot 43}(\alpha)= & 2\left(\operatorname{Dens}_{2}(\psi=1) \cdot \operatorname{Dens}_{43}\left(\varepsilon_{-43}=1\right)\right. \\
& \left.+\operatorname{Dens}_{2}(\psi=-1) \cdot \operatorname{Dens}_{43}\left(\varepsilon_{-43}=-1\right)\right) .
\end{aligned}
$$

Indeed, let us consider Theorem 19, recalling that $\mathrm{C}_{m}=1$. Let us call $H_{+}$the subset of $\mathcal{G}\left(m^{\infty}\right)$ consisting of elements whose image in $\mathcal{G}(2)$ satisfies $\psi=1$ and whose image in $\mathcal{G}(43)$ satisfies $\varepsilon_{-43}=1$ and define analogously $H_{-}$with $\psi=-1$ and $\varepsilon_{-43}=-1$. Write $H_{+}=H_{2,+} \times H_{43,+}$, where $H_{2,+} \subseteq \mathcal{G}\left(2^{\infty}\right)$ and $H_{43,+} \subseteq \mathcal{G}\left(43^{\infty}\right)$. Similarly, write $H_{-}=H_{2,-} \times H_{43,-}$. The formula of Theorem 19, considering the two contributions for Dens $_{2.43}(\alpha)$ coming from $H_{+}$and $H_{-}$, gives

$$
\operatorname{Dens}^{+}=\frac{\# \mathcal{G}(2) \# \mathcal{G}(43)}{\# \mathcal{G}(2 \cdot 43)} \int_{H_{2,+}} \frac{\mathrm{w}_{2 \infty}(M)}{\# \operatorname{ker}(M-I)} d \mu_{\mathcal{G}_{2} \infty}(M) \cdot \int_{H_{43,+}} \frac{\mathrm{w}_{43} \infty}{\# \operatorname{ker}(M-I)} d \mu_{\mathcal{G}_{43} \infty}(M),
$$

and similarly for Dens ${ }^{-}$. This yields formula (7.2).

For the point $2 \alpha$, by [4, Example 28], we only need to scale (7.1) by a factor 2, giving $1 / 3$ and $3 / 7$ as the two contributions to $\operatorname{Dens}_{2}(2 \alpha)$ by [4, Theorem 35]. For the point $4 \alpha$, we adapt (7.1) as $2 \cdot 1 / 2 \cdot 2^{-2}+\sum_{b>1} 4 \cdot 1 / 2 \cdot 2^{-2 b}$ and obtain $5 / 12$ and $13 / 28$ as the two contributions to $\operatorname{Dens}_{2}(4 \alpha)$.

ACKNOWLEDGments. The authors would like to thank Davide Lombardo and the referee for their valuable comments on the paper.

\section{REFERENCES}

1. N. Jones, Almost all elliptic curves are Serre curves, Trans. Amer. Math. Soc. 362(3) (2010), $1547-1570$.

2. R. Jones and J. Rouse, Galois theory of iterated endomorphisms, Proc. Lond. Math. Soc. (3) 100(3) (2010), 763-794. Appendix A by Jeffrey D. Achter.

3. D. Lombardo and A. Perucca, The 1-eigenspace for matrices in $\mathrm{GL}_{2}\left(\mathbb{Z}_{\ell}\right)$, New York J. Math. 23 (2017), 897-925.

4. D. Lombardo and A. Perucca, Reductions of points on algebraic groups, J. Inst. Math. Jussieu (2020), 1-33. doi:10.1017/S1474748019000598.

5. A. Perucca, Prescribing valuations of the order of a point in the reductions of abelian varieties and tori, J. Number Theory 129 (2009), 469-476.

6. J.-P. Serre, Propriétés galoisiennes des points d'ordre fini des courbes elliptiques, Invent. Math. 15(4) (1972), 259-331.

7. A. V. Sutherland, Computing images of Galois representations attached to elliptic curves, Forum Math. Sigma 4(e4) (2016), 79.

8. The LMFDB Collaboration, The L-functions and modular forms database (2016). http:// www.lmfdb.org.

9. The Sage Developers, SageMath, the Sage Mathematics Software System (Version 7.3) (2016). http://www.sagemath.org. 